\title{
Possibility of superconductivity in the repulsive Hubbard model on the Shastry-Sutherland lattice
}

\author{
Takashi Kimura ${ }^{1,2}$ 母 Kazuhiko Kuroki ${ }^{3}$, Ryotaro Arita ${ }^{1}$, and Hideo Aoki ${ }^{1}$ \\ ${ }^{1}$ Department of Physics, University of Tokyo, Hongo, Tokyo 113-0033, Japan \\ ${ }^{2}$ Department of Complexity Science and Engineering, \\ University of Tokyo, Hongo, Tokyo 113-0033, Japan \\ ${ }^{3}$ Department of Applied Physics and Chemistry, The University of Electro-Communications, Chofu, Tokyo 182-8585, Japan
}

(Dated: May 1, 2018)

\begin{abstract}
Possibility of superconductivity from electron repulsion in the Shastry-Sutherland lattice, which has a spin gap at half filling, is explored with the repulsive Hubbard model in the fluctuationexchange approximation. We find that, while superconductivity is not favored around the halffilling, superconductivity is favored around the quarter-filling. Our results suggest that the Fermi surface nesting is more important than the spin dimerization for superconductivity.
\end{abstract}

PACS numbers: PACS numbers: 74.70.Kn, 74.20.Mn

Superconductivity from electron repulsion has acquired a renewed momentum from the discovery of high$T_{C}$ cuprates [1], and the subsequent seminal proposal by Anderson [2] that the electron correlation should be at the heart of the superconductivity. An important, and still not fully understood, question dating back to the early stage along this line is the relation between the spin gap and the superconductivity. A gap in the spin excitation associated with a quantum spin liquid is, crudely speaking, favorable for a singlet-pairing formation. So one may naively expect that doping of carriers into a spin-gapped Mott insulator will show superconductivity. A clear-cut example is the $t-J$ or Hubbard model on the two-leg ladder [3], where the un-doped ladder is a spingapped Mott insulator and the system indeed becomes superconducting when doped.

On the other hand, the spin gap is obviously not a necessary condition for superconductivity. The Hubbard model on the 2D square lattice or the three-leg ladder 4] are examples, which are spin-gapless at half-filling [3, 5], but superconduct when doped[4].

If we go back to the superconductivity from repulsive electron-electron interactions in a broader context, usual understanding is that the effective attraction between electrons are mediated by spin fluctuations. [6, 7] An important difference from the superconductivity from attraction is that the effective attraction arises from pairscattering processes across which the BCS gap function changes sign, so the effective attraction is wavenumber dependent and the pairing is anisotropic (typically $d$-wave as in the cuprates). Numerically, a quantum Monte Carlo calculation that takes care of the relevant energy scale [8, 9] has shown an enhanced pairing correlation in the repulsive Hubbard model. Analytically, the fluctuation-exchange (FLEX) studies 10, 11, 12], which is a kind of the renormalized random phase approximation based on the Fermi liquid picture, have shown $d$ -

\footnotetext{
*Electronic address: kimura@cms.phys.s.u-tokyo.ac.jp
}

wave superconductivity with a transition temperature $T_{C} \sim O(0.01 t)$ for the repulsive Hubbard model. Notably, $T_{C}$ is two orders of magnitude smaller than $t$, although $T_{C}$ amounts to $\sim 100 \mathrm{~K}$ as in the high- $T_{C}$ cuprates if we take $t \sim 0.4 \mathrm{eV}$.

Recently, the multiband lattices having disconnected Fermi surfaces have been proposed as systems having much higher $T_{C}$ 's $(\gg 0.01 t)$ 14, 15, 16, 17, 18]. While the motivation for considering disconnected Fermi surfaces is to raise the $T_{C}$, which is lowered down to $\sim 0.01 t$ in ordinary lattices because the nodes in the BCS gap function intersect the Fermi surface: when the Fermi surface consists of pockets, the nodes can run in between the pockets. If we look at the proposed lattices in real space, on the other hand, we immediately notice that all of them happen to have dimerized structures in some way or other. From the spin-gap point of view one might consider this reasonable, since the dimerization can favor a spin gap when the dimerization is strong. However, we cannot identify which of the dimerization and the disconnected Fermi surface contributes to the higher $T_{C}$, since the dimerization and the disconnected Fermi surface are simultaneously satisfied in these lattices.

Now, there is an important and unresolved question of whether the spin gap associated with strong dimerization is a sufficient condition for superconductivity, i.e., whether the doped spin-gapped system can always become superconducting with an appreciable $T_{C}$. There are several lattices intensively studied from the viewpoint of the spin gap in the Mott-insulator phase. Among them is the Shastry-Sutherland (SS) lattice (Fig. 1), where we have a herringbone array of dimers. This lattice is first proposed by Shastry and Sutherland [19] about two decades ago. They found that the ground state in the Heisenberg model is spin-gapped when strongly dimerized, $J_{2} / J_{1}<0.5$, where $J_{1}\left(J_{2}\right)$ is the spin-spin interaction within (across) dimer. With the exact diagonalization method for finite clusters, Miyahara and Ueda 20] have recently obtained a more accurate critical value, $J_{2} / J_{1} \simeq 0.7$, for the appearance of the spin gap. Analytic studies such as a series expansion [21], rigorous 


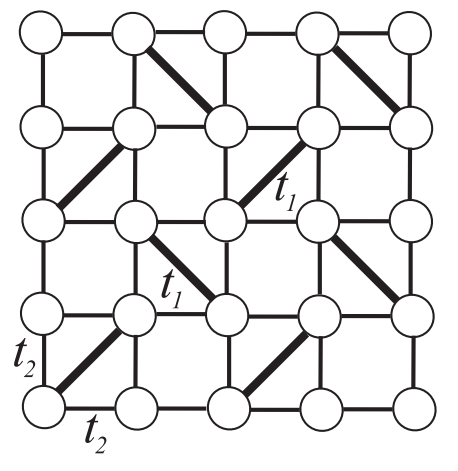

FIG. 1: The Shastry-Sutherland lattice.

bounds [22], and large- $N$ theories 23] have indicated similar boundaries.

A recent impetus came from experiments on a copper compound, $\mathrm{SrCu}_{2}\left(\mathrm{BO}_{3}\right)_{2}, 24$ where the ShastrySutherland lattice is realized. Experimental results for magnetic susceptibility [24, 25], Cu NQR 24], high-field magnetization [24], ESR [26], Raman scattering 27], inelastic neutron scattering [28] and specific heat [29] show that this compound has a dimerized ground state with a spin gap $\simeq 30 \mathrm{~K}$ with no long-range magnetic order. 30]

So the SS lattice is an appropriate system for the above question of whether a spin-gapped system can superconduct when doped. A recent study [33] for the $t-J$ model on the SS lattice has indeed discussed possible superconducting transition at low temperatures of $O(0.01 t)$ based on RVB type mean field theory 34]. Usually the SS lattice is studied with the Heisenberg model, which corresponds to the half-filled Hubbard model. Here we have opted for the Hubbard model, since we can only study a finite Hubbard $U$, but also look at the SS lattice around quarter filling, which we propose here to be interesting. The band filling controls the shape of the Fermi surface after all, so that it should be an important parameter for studying the question at hand. Quarter-filled case is not unrealistic, since the herringbone structure of the SS lattice strongly reminds us of a class of dimerized organic crystals, where the band is often quarter filled rather than half-filled.

So here we take the SS lattice to study superconductivity in the Hubbard model. We adopt the FLEX approximation, which has to be extended to four-band systems 15, 16, 17, 31] to treat the SS lattice that has four atoms per unit cell. Superconducting transition has been examined with Eliashberg's equation 32. We shall show that the Hubbard model, around half filling, does not exhibit superconductivity with significant $T_{C}$. So this provides an example in which a doped spin-gapped system does not guarantee an appreciable $T_{C}$. By contrast, the Hubbard model around the quarter-filling exhibits superconductivity with a $d$-wave pairing when the dimerization is strong $t_{2} \ll t_{1}$. We shall discuss this in terms of the shape of the Fermi surface and the pair-scattering

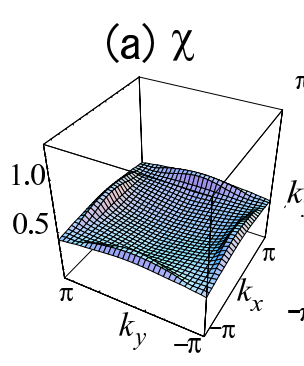

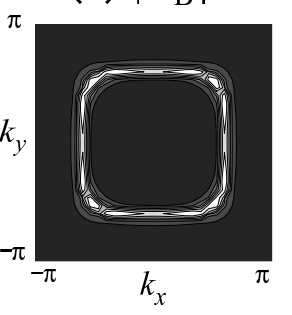

(b) $\left|G_{\mathrm{B}}\right|^{2}$ (c) $\phi_{\mathrm{B}}$

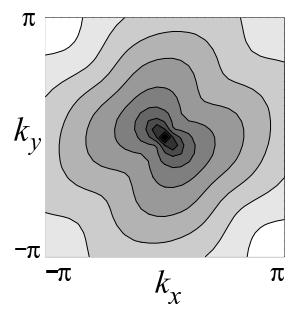

FIG. 2: Result for $\chi(\mathrm{a}),\left|G_{B}\right|^{2}$ (b), and $\phi_{B}$ (c) against $k_{x}, k_{y}$ for the lowest Matsubara frequency for the nearly half-filled $n=0.85$ with $U=7, t_{1}=1.8, t_{2}=1.0$, and $T=0.025$.

processes on it.

In the four-band version of the FLEX [15, 16, 17, 31], Green's function $G$, spin susceptibility $\chi$, self-energy $\Sigma$ are $4 \times 4$ matrices, e.g., $G_{l m}\left(\vec{k}, i \epsilon_{p}\right)$ with $\epsilon_{p}$ being the Matsubara frequency. Here $l, m$ refer to the four sites in a unit cell, which can be unitary-transformed to band indices. We obtain the eigenvalue and the superconducting gap function $\phi_{l m}$ by solving the linearized Eliashberg's equation [32]. For the susceptibility $\chi$, we quote hereafter the value of the largest component when we diagonalize its matrix. In the present study, we take $32 \times 32 k$-points and up to 8196 Matsubara frequencies, or $64 \times 64 k$-points and up to 4096 Matsubara frequencies.

We start with the case of near half-filling. Here we take the band filling $n$ (=number of electrons/number of sites) $=0.85$, along with the on-site repulsion $U=7$ and the transfer energy within (across) dimer $t_{1}= \pm 1.25$ $\left(t_{2}=1.0\right)$. This value of $t_{1}$ has been adopted in Ref. 33. as appropriate to $\mathrm{SrCu}_{2}\left(\mathrm{BO}_{3}\right)_{2}$. Corresponding Heisenberg model at half filling has a spin gap because of $J_{2} / J_{1} \sim t_{2}^{2} / t_{1}^{2} \ll 0.7$.

The maximum eigenvalue of Eliashberg's equation $\lambda$ has turned out to be much smaller than unity $(\lambda \simeq 0.43)$ at low temperature $(0.01 \leq T \leq 0.04)$. We have also calculated for $t_{1}=1.8$, which is a favorable value for the near-quarter-filled case as we shall see below, but $\lambda$ is again very small $(\lambda=0.36,0.37$ at $T=0.04,0.01$, respectively).

Figure 2 shows the spin susceptibility $(\chi)$, Green's function $(G)$ and the gap function $(\phi)$ for the second band from the bottom (called $B$, which crosses the Fermi energy) for $t_{1}=1.8$ and $t_{2}=1.0$ at $T=0.025$. We see the susceptibility has no strong peaks, unlike in the square lattice which has a large antiferromagnetic peak in the susceptibility around $\vec{k}=(\pi, \pi)$ that is relevant to the superconductivity. The weak spin structure in the SS lattice should be due to the spin gap and/or the spin frustration which strongly prevent(s) the long range spin correlation when the dimerization is strong [35].

If we weaken the dimerization by making $t_{1}$ sufficiently smaller than $t_{2}$, the system becomes superconductive. 
(a) $\chi$

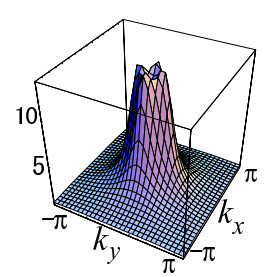

(b) $\left|G_{\mathrm{B}}\right|^{2}$

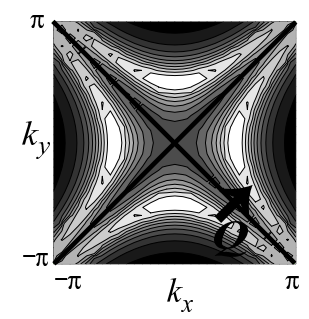

(c) $\phi_{\mathrm{B}}$

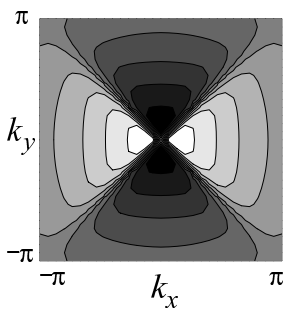

FIG. 3: The same plot as in Fig. 2 for a weaker dimerization with $t_{1}=0.5$. $\mathbf{Q}$ represent the nesting vector across $k_{x}= \pm k_{y}$ (solid lines).

Figure 3 shows the spin susceptibility, Green's function and the gap function for $t_{1}=0.5$ and $t_{2}=1.0$ at $T=0.025$, where we obtain a large $\lambda=0.94$ close to unity ( $\lambda$ becomes unity at lower temperature $T \sim 0.02$ ). In the figure we see the Fermi-surface nesting across $k_{x}= \pm k_{y}$ is appreciable and the spin susceptibility has a strong peak around $(0,0)$, which corresponds to the peak around $(\pi, \pi)$ on the square lattice $\left(t_{1}=0\right)$ folded. The result indicates that the dimerization (or the spin gap) is by no means a sufficient condition for very high $T_{C}$. If we turn to the very high $T_{C}$ systems obtained in 14, 15, 16, 17, 18], dimerization causes the disconnected Fermi surfaces accompanied by antiferromagnetic spin fluctuations. So the present result is an example in which a dimerization works unfavorably for superconductivity in a simply-connected Fermi surface. In this sense, the disconnected Fermi surfaces rather than the dimerization is essential for very high $T_{C}$ in $[1,15,16,17,18]$.

We have a drastically different situation when we change the band filling to quarter filling. Figure 4 shows the temperature dependence of $\lambda$ at $n=0.55$ with the same parameter as those at $n=0.85$. We see $\lambda$ is strongly enhanced at low temperatures.

Figure 5 shows $\chi, G$ and $\phi$ for the lowest two bands (called A and B) that cross the Fermi energy for this filling. The peak in the spin susceptibility around $(0,0)$ is much stronger than for the half-filling. The spin fluctuation should mediate the pair-scattering across which the gap function has opposite signs, resulting in $d$-wave superconductivity. How can this happen when the nesting vector is close to $(0,0)$ ? Figure $5(\mathrm{~d})$ depicts the answer: the gap function has a $d$-wave symmetry as in the square lattice near half-filling. This is physically natural because the SS lattice around quarter filling is effectively a square lattice around half filling in the strongly dimerized case $\left|t_{1}\right| \gg\left|t_{2}\right|$.

As mentioned above, it is interesting to compare the SS lattice with organic, $d$-wave superconductors such as $\kappa$-(BEDT-TTF $)_{2}$ X. 36] If we assume the dimerization is sufficiently strong, the original system around quarter filling can be represented by a two-band 37,40$]$ or a single-band 38, 39 Hubbard model around half

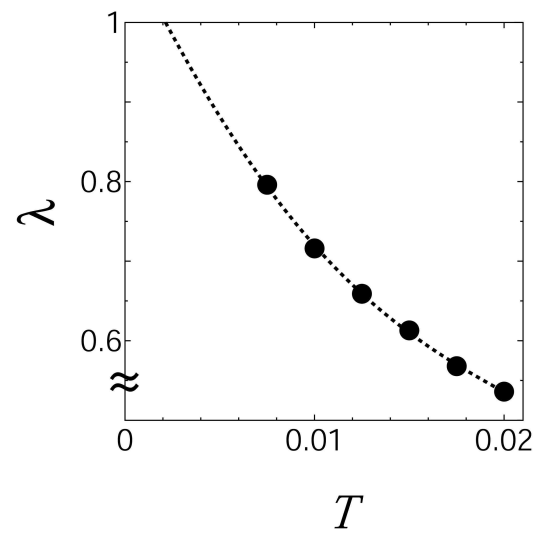

FIG. 4: For a nearly quarter-filled band $(n=0.55) \lambda$ is plotted as a function of temperature $T$ for $U=7, t_{1}=1.8$ and $t_{2}=1.0$. The dotted curve is a least-square fit by a fourth-order polynomial. Here we take $32 \times 32 k$-points and up to 8196 Matsubara frequencies. Error bars for different choice of the $k$-point mesh and Matsubara frequencies are $\sim 10 \%$ at low temperatures.

filling. 31 There, superconductivity is enhanced for $\sim$ $1 / 4$ filled band by strong dimerization as in the present study. Although organic materials that can be modeled by the SS lattice have not been known, it would be interesting to search for them.

In summary, we have studied superconductivity in the Hubbard model on the Shastry-Sutherland lattice with FLEX. Our analysis shows superconducting transition temperature, if any, is very small around half filling despite the presence of a spin gap due to the dimerization, while superconductivity is favored around quarter filling. Comparison with the RVB theory for the $t-J$ model 33. is interesting because the RVB theory has shown $T_{C}$ of $O(0.01 t)$. While this may naively seem inconsistent with our result, the $T_{C}$ of a conventional RVB theory on the SS lattice is much smaller than that $(\sim O(0.1 t))$ for the square lattice [41, 42]. So the situation about the difference between $T_{C}$ 's is similar to this case.

This work was supported in part by a Grant-in-Aid for Scientific Research and Special Coordination Funds from the Ministry of Education of Japan. Numerical calculations were performed at the Supercomputer Center, Institute for Solid State Physics, University of Tokyo. 
(a)
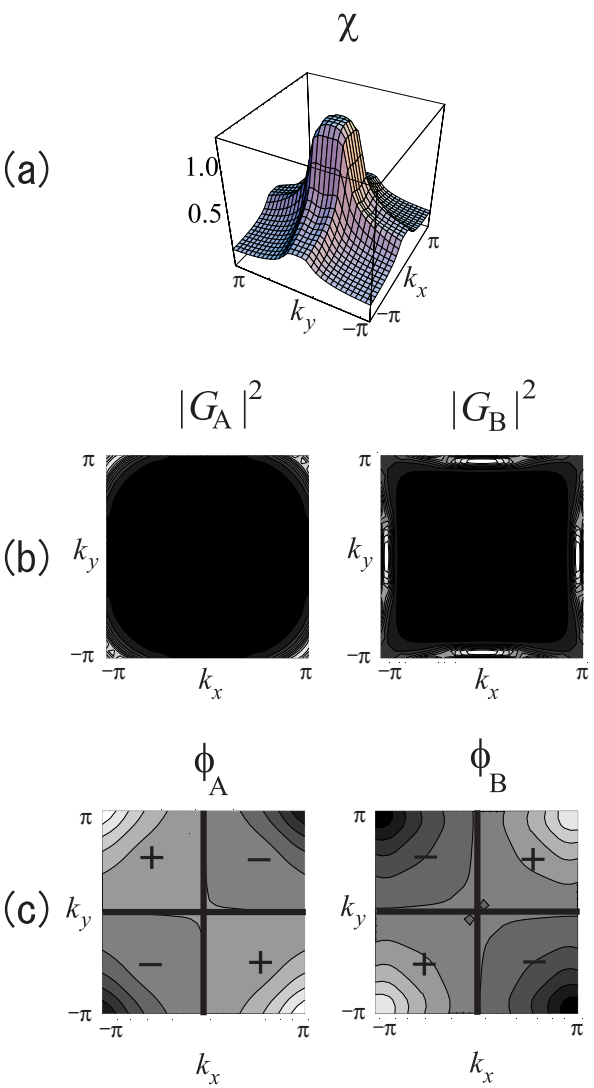

(d)

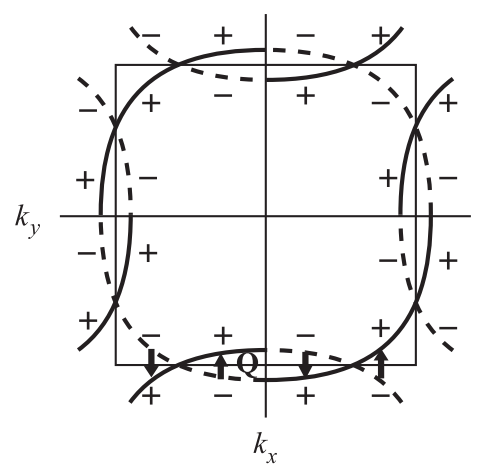

FIG. 5: For a nearly quarter-filled band $(n=0.55) \chi(\mathrm{a})$, $\left|G_{\nu}\right|^{2}(\mathrm{~b})$, and $\phi_{\nu}(\mathrm{c})(\nu=A, B)$ are plotted against $k_{x}, k_{y}$ for the static case (lowest Matsubara frequency) for $U=7$, $t_{1}=1.8, t_{2}=1.0$, and $T=0.025$. (d) schematically depicts the sign of the gap function on the Fermi surface, where $\mathbf{Q}$ and arrows represent the nesting vector $\sim(0,0)$.

[1] J.G. Bednorz and K.A. Müller, Z. Phys. B 64, 189 (1986).

[2] P.W. Anderson, Science 235, 1196 (1987).

[3] For a review, see, E. Dagotto and T.M. Rice, Science 271, 618 (1996). See also K. Kuroki, T. Kimura, and H. Aoki, Phys. Rev. B 54, R15641 (1996).

[4] T. Kimura, K. Kuroki and H. Aoki, Phys. Rev. B.
54, R9608 (1996); ibid. J. Phys. Soc. Jpn. 66, 1599 (1997) and 67, 1377 (1998); H.J. Schulz, in "Correlated Fermions and Transport in Mesoscopic Systems" ed. by T. Martin, G. Montambaux, J. Tran Thanh Van (Editions Frontières, Gif-sur-Yvette, 1996) p. 81.

[5] See, for a review, E. Manousakis, Rev. Mod. Phys. 63, 1 
(1991).

[6] See, e.g., D.J. Scalapino, E. Loh, and J.E. Hirsch, Phys. Rev. B 35, 6694 (1987); S.R. White, D.J. Scalapino, R.L. Sugar, N.E. Bickers, and R.T. Scalettar, Phys. Rev. B 39, 839 (1989).

[7] K. Ueda, T. Moriya, and Y. Takahashi in Electronic Properties and Mechanisms of High $T_{C}$ Superconductors ed. by T. Oguchi et al. (North Holland, 1992), p.145; J. Phys. Chem. Solids 53, 1515 (1992); T. Moriya and K. Ueda, Adv. in Phys. 49, 555 (2000).

[8] K. Kuroki and H. Aoki, Phys. Rev. B 56, R14287 (1997).

[9] See, for a review, H. Aoki in Recent progress in many-body theories ed. by R.F. Bishop et al. ('Advances in quantum many-body theory' Vol. 6, World Scientific, Singapore, 2002), p.13.

[10] N.E. Bickers, D.J. Scalapino, and S.R. White, Phys. Rev. Lett. 62, 961 (1989).

[11] S. Grabowski, M. Langer, J. Schmalian, and K.H. Bennemann, Europhys. Lett. 34, 219 (1996).

[12] T. Dahm and L. Tewordt, Phys. Rev. B 52, 1297 (1995).

[13] R. Arita, K. Kuroki, and H. Aoki, J. Phys. Soc. Jpn. 69, 1181 (2000); Phys. Rev. B 65, 14585 (1999).

[14] K. Kuroki and R. Arita, Phys. Rev. B 64, 024501 (2001).

[15] T. Kimura, H. Tamura, K. Kuroki, K. Shiraishi, H. Takayanagi, and R. Arita, Phys. Rev. B 66, 132508 (2002).

[16] K. Kuroki, T. Kimura, and R. Arita, Phys. Rev. B 66, 184508 (2002).

[17] T. Kimura, Y. Zenitani, K. Kuroki, R. Arita, and H. Aoki, Phys. Rev. B 66, 212505 (2002).

[18] The disconnectivity of Fermi surfaces is shown to enhance $T_{C}$ in a three-dimensional system as well [S. Onari, K. Kuroki, R. Arita, and H. Aoki, preprint].

[19] B.S. Shastry and B. Sutherland, Physica 108B, 1069 (1981).

[20] S. Miyahara and K. Ueda, Phys. Rev. Lett. 82, 3701 (1999).

[21] A. Koga and N. Kawakami, Phys. Rev. Lett. 84, 4461 (200); C. Knetter, A. Bühler, E. Müller-Hartmann, and G.S. Uhrig, Phys. Rev. Lett. 85, 3958 (2000); Z. Weihong, J. Oitmaa and C. Hamer, Phys. Rev. B 65, 014408 (2001).

[22] U. Löw and E. Müller-Hartmann, J. Low Temp. Phys. 126, 1135 (2002); ibid 127, 290 (2002).

[23] C. Chung, J. Marston, and S. Sachdev, Phys. Rev. B 64, 134407 (2001).

[24] H. Kageyama, K. Yoshimura, R. Stern, N.V. Mushnikov, K. Onizuka, M. Kato, K. Kosuge, C.P. Slichter, T. Goto, and Y. Ueda, Phys. Rev. Lett. 82, 3168 (1999).

[25] H. Kageyama, K. Onizuka, T. Yamauchi, Y. Ueda, S. Hane, H. Mitamura, T. Goto, K. Yoshimura, and K. Kosuge, J. Phys. Soc. Jpn. 68, 1821 (1999).

[26] H. Nojiri, H. Kageyama, K. Onizuka, Y. Ueda, and M. Motokawa, J. Phys. Soc. Jpn. 68, 2906 (1999).

[27] P. Lemments, M. Grove, M. Fisher, G. Güntherodt, V.N. Kotov, H. Kageyama, K. Onizuka, and Y. Ueda, Phys. Rev. Lett. 85, 2605 (2000).

[28] H. Kageyama, M. Nishi, N. Aso, K. Onizuka, T. Yosi- hama, K. Nukui, K. Kodama, K. Kakurai, and Y. Ueda, Phys. Rev. Lett. 84, 5876 (2000).

[29] H. Kageyama, H. Suzuki, M. Nohara, K. Onizuka, H. Takagi, and Y. Ueda, Physica B 281-282, 667 (2000).

[30] Magnetization plateaus in finite magnetic fields have been experimentally observed by $\mathrm{K}$. Onizuka, $\mathrm{H}$. Kageyama, Y. Narumi, K. Kindo, Y. Ueda, and T. Goto [J. Phys. Soc. Jpn. 69, 1016 (2000)] as another striking property in this compound, and has been theoretically examined by Y. Fukumoto, J. Phys. Soc. Jpn. 70, 1397 (2001); T. Momoi and K. Totsuka, Phys. Rev. B 62, 15067 (2000); G. Misguich, Th. Jolicoeur, and S. Girvin, Phys. Rev. Lett. 87, 097203 (2001); A. Fledderjohann and K.-H. Mütter, Phys. Rev. B 65, 212406 (2002).

[31] To be precise, the pairing symmetry should be determined by the original four-band model as shown by $\mathrm{K}$. Kuroki, T. Kimura, R. Arita, Y. Tanaka, and Y. Matsuda [Phys. Rev. B 65, 100516 (2002)].

[32] G.M. Eliashberg, Zh. Eksp. Teor. Fiz. 38, 996 (1960) [Soviet Phys. JETP 11, 696 (1960)].

[33] B.S. Shastry and B. Kumar, cond-mat/0204228 (unpublished).

[34] See, e.g., G. Baskaran, Z. Zou and P.W. Anderson, Solid State Commun. 63, 973 (1987); Y. Suzumura, Y. Hasegawa, and H. Fukuyama, J. Phys. Soc. Jpn. 57, 401 (1988); G. Kotliar and J. Liu, Phys. Rev. B 38, 5142 (1988).

[35] Generally, it is hard to identify which condition is essential for preventing the long-range spin correlation. For example, the spin frustration prevents the spin correlation in many systems but the weak next-nearest hopping in the square lattice does not strongly prevents the spin correlation and even enhances the superconductivity [38]. On the other hand, the spin gap also prevents the spin correlation in many systems such as ladder systems, but does not result in very high $T_{C}$ systems.

[36] For a review, see, R.H. Mckenzie, Science 278, 820 (1997); see also K. Kanoda, Physica C 282-287, 299 (1997).

[37] J. Schmalian, Phys. Rev. Lett. 81, 4232 (1998).

[38] H. Kino and H. Kontani, J. Phys. Soc. Jpn. 67, 3691 (1998).

[39] H. Kondo and T. Moriya, J. Phys. Soc. Jpn. 67, 3695 (1998); T. Jujo, S. Koikegami, and K. Yamada, J. Phys. Soc. Jpn. 68, 1331 (1999); K. Kuroki and H. Aoki, Phys. Rev. B 60, 3060 (1999).

[40] K. Kuroki and H. Aoki, Phys. Rev. B 60, 3060 (1999).

[41] G. Kotliar and J. Liu, Phys. Rev. B 38, 5142 (1988); Y. Suzumura, Y. Hasegawa, and H. Fukuyama, J. Phys. Soc. Jpn. 57, 401 (1988); M.U. Ubbens and P.A. Lee, Phys. Rev. B 46, 8434 (1992).

[42] One might consider that the $t-J$ model shows too high $T_{C}$ compared with those of cuprates. However, M.U. Ubbens and P.A. Lee [Phys. Rev. B 49, 6853 (1994)] showed that the superconducting $T_{C}$ on the square lattice is suppressed as $T \sim O(0.01 t)$, if one takes a fluctuating gauge field into account. 